\title{
Primary Human Immunodeficiency Virus Infection and Rhabdomyolysis
}

\author{
Abeer Moanna ${ }^{1,2}$, Jacek Skarbinski ${ }^{1}$, Ameeta S Kalokhe ${ }^{1}$, David Rimland ${ }^{1,2}$ and Nadine G. Rouphael ${ }^{1}$
}

${ }^{1}$ Division of Infectious Disease, Emory University school of Medicine, USA

${ }^{2}$ Atlanta VA Medical Center, GA, USA

\begin{abstract}
Objectives: Over the past few years we have seen 3 patients with primary HIV infection and rhabdomyolysis in our institution.

Methods: We report our 3 cases in addition to the review of the English-language literature for all reported cases of rhabdomyolysis and primary HIV infection.

Results: In addition to our 3 cases we found 11 cases of primary HIV infection presenting with rhabdomyolysis.

Conclusion: In patients presenting with rhabdomyolysis and no obvious precipitating factor, primary HIV infection should be included on the differential count.
\end{abstract}

Keywords: Human immunodeficiency virus (HIV); Primary HIV infection (PHI); Rhabdomyolysis

Rhabdomyolysis is a syndrome characterized by skeletal muscle breakdown, myoglobinuria, and creatine phosphokinase (CPK) elevation and can frequently lead to renal dysfunction. Numerous precipitating factors have been linked to rhabdomyolysis including trauma, seizures, drugs, toxins, metabolic derangements, severe exercise, and infections [1,2]. Human immunodeficiency virus (HIV) infection has been associated with rhabdomyolysis $[1,3,4]$. Whether HIV itself or a different agent such as another infection or a drug is the direct cause of rhabdomyolysis is not clearly understood. We present 3 cases of Primary HIV infection (PHI) and rhabdomyolysis from our institution. In addition, we include a review of the English-language literature of reported cases of PHI and rhabdomyolysis and discuss the importance of recognizing rhabdomyolysis as a presenting illness in patients with PHI.

\section{Case Presentation}

Case 1: A 27 year old African American male presented to the emergency room with pain in both legs, nausea, vomiting, and diarrhea. His past medical history was significant for an episode of proctitis 2 years prior to his presentation. He denied taking regular medications and reported occasional alcohol and marijuana use. On physical exam, he was afebrile at $36.9^{\circ} \mathrm{C}$. He had right upper quadrant tenderness and moderate tenderness on palpation of both legs with no associated swelling or erythema. His laboratory data were significant for a serum creatinine of $1.9 \mathrm{mg} / \mathrm{dl}$ (normal $0.5-1.2 \mathrm{mg} / \mathrm{dl}$ ), an aspartate aminotransferase (AST) of $663 \mathrm{IU} / \mathrm{L}$ (normal 8-40 IU/L), an alanine aminotransferase (ALT) of 313 IU/L (normal 0-44 IU/L), and a CPK of $12888 \mathrm{U} / \mathrm{L}$ (normal 21-160 U/L). Hepatitis B and C serologies, Hepatitis A IgM, a monospot test, and a plasma Cytomegalovirus (CMV) DNA polymerase chain reaction (PCR) test were all negative. In addition, blood alcohol level and acetaminophen level were both undetectable. A urine drug screen (UDS) that looks for amphetamines, barbiturates, benzodiazepines, opiates, marijuana, and cocaine was performed and was negative. He had an HIV antibody ELISA test done 3 days prior to his presentation at a different facility and that was negative. An HIV1 RNA PCR was sent and the patient was treated with intravenous hydration and alkanization of his urine with a good response. The HIV1 RNA viral load was reported as $>750,000$ few days after the patient was discharged from the hospital. Two weeks later, a repeat blood draw was sent for an HIV antibody ELISA test and HIV western blot and both were positive.

Case 2: A 19 year old African American male presented to the hospital with fever, sore throat, myalgias, arthralgias, decreased urine output, and dark urine for few days. He had no significant past medical history, denied taking any regular medications, and reported no alcohol, tobacco, or illicit drug use. He was a full-time student who lived at home with his parents. He reported having unprotected receptive and insertive anal intercourse two weeks prior to admission. On physical examination, he was febrile to $38.9^{\circ} \mathrm{C}$, had oral mucositis and thrush, and muscle tenderness. His laboratory data was significant for a serum creatinine of $8.0 \mathrm{mg} / \mathrm{dL}$. In addition, he had a CPK of $>16,000 \mathrm{IU} / \mathrm{L}$, an ALT of $886 \mathrm{IU} / \mathrm{L}$ and an AST of $3963 \mathrm{IU} / \mathrm{L}$, and a urinalysis that revealed protein and blood in the urine. Hepatitis $\mathrm{B}$ and $\mathrm{C}$ serologies, Hepatitis A IgM, Epstein-Barr virus (EBV) serologies, and plasma CMV DNA PCR were all negative. In addition, he had Streptococcus mitis bacteremia. His HIV antibody ELISA test was weakly positive and an HIV western blot was indeterminate with only a p24 band. His HIV1 viral load and his CD4 count were $>750,000$ copies $/ \mathrm{ml}$ and $34(3 \%)$ respectively and was diagnosed with $\mathrm{PHI}$. He required hemodialysis during his hospitalization and completed a course of intravenous penicillin $\mathrm{G}$ for his bacteremia. His CPK decreased to $1871 \mathrm{IU} / \mathrm{L}$ on the day of discharge from the hospital. He recovered his renal function after 6 weeks. A repeat HIV antibody ELISA test sent 2 weeks later was positive with an HIV-1 RNA viral load of 297,000 copies/ml.

Case 3: A 40 year old male presented to the emergency room with a 2 week history of fevers and diffuse myalgias. His past medical history was significant for sleep apnea, allergic rhinitis, and gonococcal and

*Corresponding author: Nadine Rouphael, 603 Church Street, Decatur, GA 300330, USA, Tel: 404-822-141; Fax: 404-377-6962; E-mail: nroupha@emory.edu

Received February 16, 2011; Accepted March 18, 2011; Published March 19 2011

Citation: Moanna A, Skarbinski J, Kalokhe AS, Rimland D, Rouphael NG (2011) Primary Human Immunodeficiency Virus Infection and Rhabdomyolysis. J AIDS Clinic Res 2:119. doi:10.4172/2155-6113.1000119

Copyright: ( $) 2011$ Moanna A, et al. This is an open-access article distributed under the terms of the Creative Commons Attribution License, which permits unrestricted use, distribution, and reproduction in any medium, provided the original author and source are credited. 
chlamydial urethritis. Over the preceding month he had unprotected sexual intercourse with two different male partners. He denied recent foreign travel, tobacco and recreational drug use but he drank 4-5 glasses of wine per week. His only medication was occasional Tylenol. On physical exam, he was febrile to $38.2^{\circ} \mathrm{C}$ and the rest of his exam was otherwise unremarkable. Laboratory data revealed a creatinine and a CPK of $1.4 \mathrm{mg} / \mathrm{dl}$ and $1176 \mathrm{IU} / \mathrm{L}$ respectively. CPK was not checked until 5 days after admission, during which period the patient had received intravenous hydration. Hepatitis A, B, C and EBV serologies were negative and an RPR was non-reactive. An HIV antibody ELISA test was negative but an HIV-1 RNA viral load and a CD4 T cell count were $>750,000$ copies $/ \mathrm{ml}$ and 189 respectively. Patient was lost to follow up after discharge from the hospital.

\section{Discussion and Literature Review}

A number of infections have been associated with rhabdomyolysis, but viruses, especially influenza A and B, are by far the most common causes [2,3]. Other viruses include HIV, enteroviruses, EBV, CMV, adenovirus, herpes simplex virus, Varicella-zoster virus, and parainfluenza virus [3]. Among bacterial agents causing rhabdomyolysis, Legionella sp. is reported as the most common cause $[2,3]$. Other bacteria that may cause rhabdomyolysis include but are not limited to Francisella sp, Streptococcus pneumoniae, Salmonella sp, and Staphylococcus aureus [2,3] .

HIV-infected patients may develop a variety of muscular disorders such as polymyositis, inclusion-body myositis, myopathy secondary to HIV therapy, HIV wasting syndrome, and rhabdomyolysis [5]. Rhabdomyolysis can occur at any stage of HIV infection [1] and is usually multifactorial [4]. Substance abuse, alcohol, infection, and drugs are among the most common precipitating factors in HIV-infected patients [4]. Raltegravir, an integrase inhibitor approved for treatment of HIV infection and used in combination with other antiretroviral drugs has been associated with rhabdomyolysis [6-8]. The exact pathogenic role of HIV itself in causing rhabdomyolysis has not been elucidated. Chariot et al speculated that skeletal muscle involvement in HIV patients could be due to immune-mediated mechanisms activated by HIV itself which lead to infiltration of the muscle by inflammatory cells leading to muscle injury and breakdown [1]. Another possible explanation is the direct invasion of the muscle by HIV itself but HIV particles have not been found in muscle fibers in most of the studies done [9]. One study using in situ hybridization and PCR methods on muscle biopsies of HIV-infected patients who had polymyositis found HIV RNA in the lymphoid cells surrounding the muscle fibers but not in the muscle itself [10].
Patients with PHI can be asymptomatic or can present with a fever, sore throat, fatigue, and weight loss [11]. Myalgias are common [11,12] but rhabdomyolysis is not a typical presentation. Other presenting signs and symptoms may include pharyngitis, lymphadenopathy, oral or genital ulcers, and aseptic meningitis [11].Laboratory abnormalities can include lymphopenia, thrombocytopenia, and elevated hepatic transaminases [13].

We searched medline through PubMed using the words rhabdomyolysis, primary HIV infection and acute HIV infection to look for cases of rhabdomyolysis and PHI in the English-language literature. Several case reports presented cases of patients with $\mathrm{PHI}$ and rhabdomyolysis $[9,12,14-22]$. A summary of these case reports in addition to our three cases is found in (Table 1) Most of the patients complained of myalgias and had a history of elevated temperatures or a fever on presentation. Nearly half of the patients reported sore throat on presentation but only a few had lymphadenopathy on physical exam. Some patients had mainly gastrointestinal symptoms such as nausea, vomiting, and diarrhea. All patients had elevated CPK on presentation and most of them had elevated AST and ALT levels when performed. Most patients had an elevated serum creatinine and few patients developed severe acute renal failure [12] including our second case. However, most patients recovered their kidney function with intravenous hydration. Most patients in these case reports had serological testing for viral infections that could contribute to their presentation with rhabdomyolysis but some were not tested for CMV One of the case reports described a patient with simultaneous primary HIV and CMV coinfection presenting with rhabdomyolysis [16]. In addition, a number of these reports did not mention if a drug screen was done and an alcohol history was not clear or an alcohol blood level was not obtained. It is noteworthy that few patients presented with rhabdomyolysis in the setting of multi-organ involvement such as liver failure [20] or myocarditis [15,20,21] in the setting of PHI. All patients including the ones from the present study survived the acute presentation of rhabdomyolysis and none was started on antiretroviral therapy immediately.

Whether HIV itself is the direct cause of rhabdomyolysis in these patients is not clear. A major limitation of the cases reported in this article or in the reports found during literature review is that not all patients had viral serologies and drug screens done to exclude a viral or a drug related rhabdomyolysis. However, we believe that it is important to include acute HIV seroconversion in the differential diagnosis when patients present with rhabdomyolysis. This is especially important since many of these patients presented with rhabdomyolysis without other symptoms that are suggestive of PHI.

\begin{tabular}{|l|l|l|l|l|l|l|}
\hline$[$ Reference] & Age/sex & Symptoms & CPK peak, U/L & AST & Remarks \\
\hline$[14]$ & $18 \mathrm{M}$ & Sore throat, red urine, myalgias, pharyngitis, and LN & 5750 & 165 & No urine drug screen performed \\
\hline$[12]$ & $29 \mathrm{M}$ & Sore throat, fever, pharyngitis, and LN & 4224 & 250 & No urine drug screen performed \\
\hline$[15]$ & $42 \mathrm{M}$ & Myalgias, Abdominal pain, and diarrhea & 63800 & 1480 & No urine drug screen performed \\
\hline$[16]$ & $20 \mathrm{M}$ & Myalgias, fever, pharyngitis, and LN & 11016 & 462 & Acute HIV/CMV coinfection \\
\hline$[17]$ & $51 \mathrm{M}$ & Myalgias, emesis, and fever & 32720 & 875 & CMV not excluded \\
\hline$[18]$ & $33 \mathrm{M}$ & Sore throat, rash, myalgias, emesis, and fever & 18840 & 321 & Survived \\
\hline$[19]$ & $46 \mathrm{M}$ & Myalgias, congestion, and fever & 5638 & 122 & Survived \\
\hline$[20]$ & $16 \mathrm{~F}$ & Myalgias, diarrhea, and fever & 2734 & 4266 & No urine drug screen performed \\
\hline$[21]$ & $19 \mathrm{~F}$ & Myalgias, rash, emesis, and fever & 10681 & 153 & CMV not excluded \\
\hline$[9]$ & $20 \mathrm{M}$ & Sore throat, abdominal pain, diarrhea, emesis, and fever & 2968 & 266 & No urine drug screen performed \\
\hline$[22]$ & $26 \mathrm{M}$ & Myalgias, sore throat, and fever & 41000 & 826 & CMV not excluded, no urine drug screen performed \\
\hline Present study & $27 \mathrm{M}$ & Myalgias, abdominal pain, diarrhea, and emesis & 12888 & 788 & Survived \\
\hline Present study & $19 \mathrm{M}$ & Sore throat, myalgias, and fever & $>50000$ & 3969 & CMV not excluded \\
\hline Present study & $40 \mathrm{M}$ & Myalgias, and fever & 11761 & 499 & CMV not excluded \\
\hline
\end{tabular}

Table 1: Case reports of patients with $\mathrm{PHI}$ presenting with rhabdomyolysis in the English-language literature. 
Citation: Moanna A, Skarbinski J, Kalokhe AS, Rimland D, Rouphael NG (2011) Primary Human Immunodeficiency Virus Infection and Rhabdomyolysis. J AIDS Clinic Res 2:119. doi:10.4172/2155-6113.1000119

In summary, HIV infection has been associated with rhabdomyolysis. Other precipitating factors include other infections, substance abuse, or medications. Clinicians need to be aware of the possibility of acute HIV seroconversion in the setting of rhabdomyolysis especially if patients have no other risk factors for rhabdomyolysis. The exact role of HIV itself in the pathogenesis of rhabdomyolysis is still unclear. Treatment of rhabdomyolysis in the setting of PHI remains supportive.

\section{References}

1. Chariot P, Ruet E, Authier FJ, Levy Y, Gherardi R (1994) Acute rhabdomyolysis in patients infected by human immunodeficiency syndrome. Neurology 44 : $1692-1696$.

2. Luck RP, Verbin S (2008) Rhabdomyolysis: a review of clinical presentation, etiology, diagnosis, and management. Pediatr Emerg Care 24: 262-268.

3. Singh U, Scheld WM (1996) Infectious etiologies of rhabdomyolysis: three case reports and review. Clin Infect Dis 22: 642-649.

4. Joshi MK, Liu HH (2000) Acute rhabdomyolysis and renal failure in HIV-infected patients: risk factors, presentation, and pathophysiology. AIDS Patient Care STDS 14: $541-548$

5. Authier FJ, Chariot P, Gherardi RK (2005) Skeletal muscle involvement in human immunodeficiency syndrome (HIV)-infected patients in the era of highly active antiretroviral therapy (HAART). Muscle Nerve 32: 247-260.

6. Zembower TR, Gerzenshtein L, Coleman K, Palella FJ Jr (2008) Severe rhabdomyolysis associated with raltegravir use. AIDS 22: 1382-1384.

7. Masia M, Enriquez R, Sirvent A, Gutierrez F (2010) Severe acute renal failure associated with rhabdomyolysis during treatment with raltegravir. A call for caution. J Infect 61: 189-190.

8. Dori L, Buonomini AR, Viscione M, Sarmati L, Andreoni M (2010) A case of rhabdomyolysis associated with raltegravir use. AIDS 24: 473-475.

9. Douvoyiannis M, Litman N (2009) Acute encephalopathy and multi-organ involvement with rhabdomyolysis during primary HIV infections. Int J Infect Dis 13: e299-304.
10. Leon-Monzon M, Lamperth L, Dalakas MC (1993) Search for HIV proviral DNA and amplified sequence in the muscle biopsies of patients with HIV polymyositis. Muscle Nerve 16: 408-413.

11. Schacker T, Collier AC, Hughes J, Shea T, Corey L (1995) Clinical and epidemiologic features of primary HIV infection. Ann Intern Med 125: 257-64.

12. del Rio C, Soffer O, Widell JL, Judd RL, Slade BA (1990) Acute human immunodeficiency virus syndrome temporally associated with rhabdomyolysis, acute renal failure, and nephrosis. Rev Infect Dis 12: 282-5.

13. Kahn JO, Walker BD (1998) Acute human immunodeficiency virus type 1 infection. N Engl J Med 339: 33-39.

14. Mahe A, Bruet A, Chabin E, Fendler JP (1989) Acute rhabdomyolysis coincident with primary HIV-1 infection. Lancet 2: 1454-1455.

15. Guillaume MP, Van Beers D, Delforge ML, Devriendt J, Cogan E (1995) Primary human immunodeficiency virus infection presenting as myopericarditis and rhabdomyolysis. Clin Infect Dis 21: 451-452.

16. Ragnaud JM, Tahbaz A, Buisson M, Morlat P, Gin H, et al. (1995) Primary infection with human immunodeficiency virus and cytomegalovirus presenting as acute rhabdomyolysis. Clin Infect Dis 20: 1077-1078.

17. Rastegar D, Claiborne C, Fleisher A, Matsumoto A (2001) A patient with primary human immunodeficiency virus infection who presented with acute rhabdomyolysis. Clin Infect Dis 32: 502-504.

18. McDonagh CA, Holman RP (2003) Primary human immunodeficiency virus type 1 infection in a patient with acute rhabdomyolysis. South Med J 96: 1027 1030.

19. Delo D, Brett AS, Postic B (2006) Primary HIV infection presenting with rhabdomyolysis. Am J Med Sci 332: 46-47.

20. Tattevin P, Camus C, Arvieux C, Ruffault A, Michelet C (2007) Multiple organ failure during primary HIV infection. Clin Infect Dis 44: e28-29.

21. Pano-Pardo JR, Alcaide ML, Abbo L, Dickinson G (2009) Primary HIV infection with multisystemic presentation. Int J Infect Dis 13: e177-180.

22. Johnson KJ, Jouett B, Ivonye CC (2011) Acute rhabdomyolysis with no apparent cause. Infect Dis Clin Pract 19: 68-69. 